Part of Journal of Research of the National Bureau of Standards, Volume 19, August 1937

\title{
DEVICE FOR TESTING HAEMACYTOMETERS AND OTHER PIPETTES OF SMALL CAPACITY
}

\author{
By Elmer L. Peffer
}

ABSTRACT

An instrument employing a volumetric method and used in the rapid and accurate testing of dilution pipettes is described. A double-walled evacuated cylinder is fitted with two metal plungers actuated by micrometer heads and a third opening is fitted with a rubber stopper having a hole to receive and hold without leakage of liquid the pipette to be tested. The test liquid is a 3 to 5 percent (by weight) solution of potassium dichromate in water. The plungers have cross-sectional areas in the ratio of $10: 1$ and have diameters designed to cover the range of all ordinary dilution pipettes.

The pipette, in the inverted position, is placed in the hole of the rubber stopper, and the large plunger, actuated by the micrometer head, is turned into the chamber until the liquid reaches the first graduation. The micrometer is then read. This micrometer is further advanced until the liquid fills the bulb and reaches the first graduation above the bulb. The micrometer is again read. The difference of these readings indicates the advancement of plunger in the test liquid. The same procedure is followed with the small plunger completing the calibration of the graduated capillary. The dilution ratios of the pipette are determined directly by the linear movement of the cylindrical plungers, as determined by the micrometer readings.

For many years it was customary practice at the National Bureau of Standards to use a weight method in the test of dilution pipettes, to determine the relative capacities, and thence the volume ratios, of the intervals tested.

In recent years, however, the great increase in the number of dilution pipettes submitted to the Bureau for test has made it necessary to develop a test method that would be more rapid than the weight method and at the same time sufficiently accurate for the purpose. With this end in view, the apparatus described herein was developed. It has been found entirely satisfactory with respect to both speed and accuracy. The apparatus is shown in figures 1 and 2 .

The principle involved is that of displacing a volume of liquid by two cylindrical plungers of known relative areas of cross section, each plunger being actuated by a calibrated micrometer screw with a graduated indicating head. The cross-sectional areas of the plungers are in the ratio of $10: 1$, and the micrometer screws and graduated heads, which actuate the plungers and indicate their positions, are of equal pitch and are graduated in the same manner. Thus, when the two plungers are advanced the same distance as indicated by the graduated micrometer heads, the volume of liquid displaced by the larger plunger is 10 times that displaced by the smaller one. The micrometer heads are of the ordinary type used by machinists and instrument makers, having a screw of 40 threads to the inch, a rotatable thimble with 25 graduations around its circumference, and a range 
of 1 inch. Thus, 1 division is equal to $0.001 \mathrm{inch}$, and readings are made to 0.0001 inch.

The large and the small cylindrical plungers have diameters of 0.3921 inch and $0.1240 \mathrm{inch}$, respectively. A movement of the full range of 1 inch, therefore, results in a liquid displacement of 0.1208 cubic inch for the larger cylinder, and 0.01208 cubic inch for the smaller. Expressed in milliliters, these volumes are approximately 2.0 and $0.2 \mathrm{ml}$, respectively. These volumes are adequate to cover the capacity range of all ordinary dilution pipettes.

Since the diameters of the cylindrical plungers are known, and the micrometer screws and graduated heads can be accurately calibrated, it would be possible to prepare a table for each plunger and micrometer showing the volumes of displaced liquid represented by various differences in micrometer readings. For use in the testing of dilution pipettes, however, such a table is unnecessary since all that is required in the testing of these pipettes is to determine the ratio of the volumes of the various intervals under test. These ratios are determined directly by the linear movement of the cylindrical plungers, as determined by the micrometer readings.

In the upper surface of the double-walled glass container is a third opening fitted with a rubber stopper having a hole of suitable size to receive and hold without leakage of liquid the pipette to be tested.

The test liquid employed is a 3 to 5 percent solution (by weight) of potassium dichromate in water. A suitable container for the test liquid is provided in the form of a double-walled evacuated glass cylinder with rounded ends, this container being mounted on a wooden base. In each end of the cylindrical container is an opening through which the micrometer plunger enters. The micrometer is sealed securely in place by a metal-in-glass ground joint.

The double-walled evacuated chamber for containing the test liquid was found necessary to prevent excessive volume changes of the test liquid as a result of temperature changes during test of a pipette.

The use of dichromate solution as the test liquid was found to have two advantages: first, this liquid served as a rust inhibitor, and second, the amber color of the liquid made possible quick and accurate setting of the liquid meniscus on the graduation lines.

In testing a dilution pipette the pipette is inverted and what is normally the upper end of the capillary tube is inserted in the hole in the rubber stopper which is in the opening in the upper surface of the double-walled glass chamber.

With the micrometer which actuates the smaller of the two cylindrical plungers set on zero, and the surface of the test liquid below the first graduation mark on the pipette, the micrometer which actuates the larger plunger is turned and the cylinder advanced into the chamber until the liquid surface in the capillary reaches the first, or lowest graduation mark. The micrometer is then read. The same micrometer is then further advanced and the test liquid forced into the pipette, filling the capillary below the mixing chamber, the mixing chamber, and the capillary up to the first graduation mark above the mixing chamber. The micrometer is then again read. The difference between the two readings of this micrometer indicates the amount the plunger has been advanced into the test liquid. 


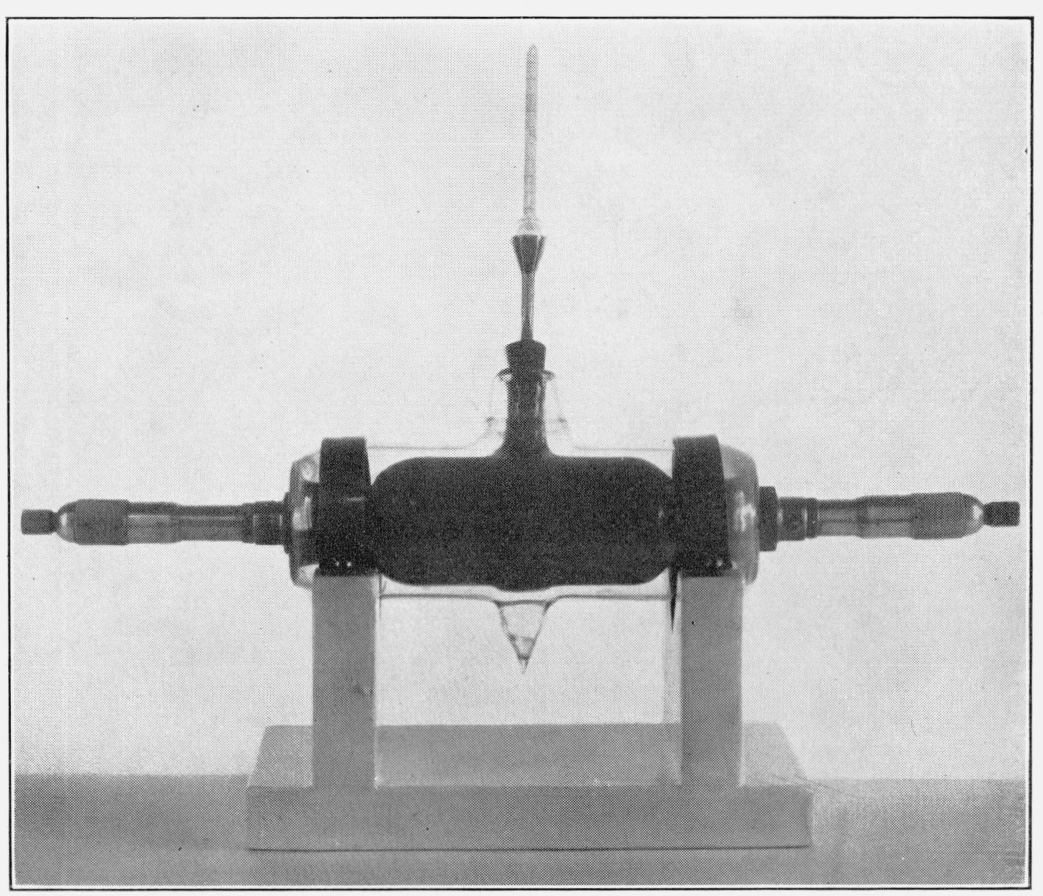

Figure 1. 
The micrometer which actuates the smaller of the two plungers is then turned in such a direction as to advance this plunger into the test liquid, and the liquid is thus forced into the pipette until the liquid surface advances in the graduated capillary tube to the desired graduation. The micrometer is then read, and, since the areas of cross section of the plungers are as 10:1, the ratio of the intervals under test is 10 times the ratio of advance of the large plunger to the advance of the small plunger, as indicated by the micrometers.
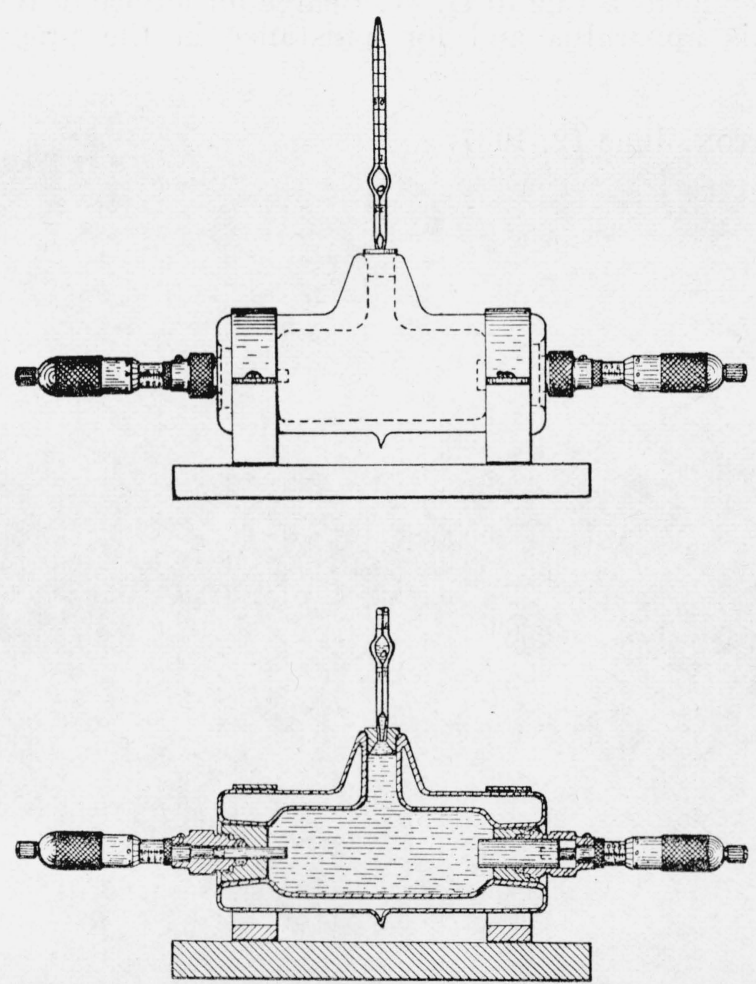

Figure 2.-Sketch and cross section of dilution pipette tesing apparatus.

The ratio of the volumes of the two intervals on the pipette, as thus determined, is the "dilution ratio" of the pipette. It is customary to determine the "dilution ratio" for each pipette when using a full unit and when using a half unit of blood; for example, 100:1 and 200:1 for pipettes used in counting red corpuscles, and 10:1 and 20:1 for those used in counting white corpuscles.

The apparatus is checked from time to time by comparison of its results with those obtained by the weight method.

In general, the tolerances permitted on dilution pipettes are 5 percent for the pipette used in counting red corpuscles and 3.5 percent for the pipette used in counting white corpuscles. The apparatus and test method herein described yield results that are accurate to about one-fifth of the tolerance. 
While the apparatus herein described was designed and is used primarily for the testing of dilution pipettes, it could readily be used for testing any other pipettes or similar apparatus within its capacity range, for the graduation and calibration of small measuring pipettes, for the filling of ampules, or for other purposes where the accurate delivery of small volumes or the testing of small capacities is desired.

Acknowledgment is due to H. W. Bearce for advice in the development of this apparatus and for assistance in the preparation of the paper.

Washington, June 12, 1937. 